\title{
Abrechnung Herzdiagnostik mit MRT und CT nach GOÄ
}

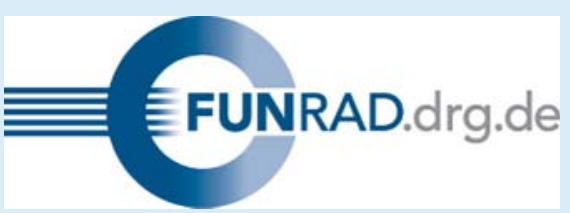

Die Untersuchungen des Herzens mit modernen Schnittbildverfahren sind weder in der GOÄ noch im EBM bisher adäquat abgebildet. Im Rahmen der Arbeiten zur Novellierung beider Gebührenordnungen hat der BDR natürlich konkrete Leistungslegenden und Bewertungsvorschläge vorgelegt. Angesichts der derzeitigen politischen Großwetterlage ist aber nicht absehbar, wann umfangreiche Neuerungen dazu tatsächlich umgesetzt werden können. Im Bereich der GKV gilt es dazu außerdem, zunächst die Frage der Herzbildgebung als Kassenleistung grundsätzlich zu klären. Nach wie vor ist der BDR der Ansicht, dass die MRT des Herzens keine Kassenleistung ist.

Vor diesem Hintergrund hat der BDR mit der DRG, der AG Herz- und Gefäßdiagnostik und FuNRad eine gemeinsame Empfehlung zur Abrechnung der Herzuntersuchungen mit CT und MRT abgestimmt. Die Empfehlung beschränkt sich auf die Darstellung der regelmäßig abrechenbaren Gebührenordnungspositionen und der im Einzelfall zusätzlich erforderlichen bzw. abrechenbaren Leistungen.

Die Frage der Gebührenhöhe ist im Einzelfall durch Bestimmung des geeigneten Steigerungsfaktors zu bestimmen ( $§ 5$ GOÄ). Dazu lassen sich einheitliche Vorgaben nicht machen. Zu beachten ist dabei aber der begrenzte Steigerungsrahmen für die Pulsoximetrie mit maximal 1,8-fach und der verbindliche Einfachsatz für die Ziffern 5377, 5732 und 5733.
Die MRT des Herzens in Ruhe und mit pharmakologischem Stress ist in derselben Sitzung nicht nebeneinander abrechenbar. Faktisch liegen hier zwar zwei vollständige Untersuchungen vor, die aber wegen der Bestimmung in den Allgemeinen Bestimmungen zu Kapitel O Ziffer I.7 und III je Sitzung nur einmal berechnungsfähig sind. Dem deutlich erhöhten Aufwand kann aber durch Steigerung auf den Höchstsatz der jeweiligen GOPen Rechnung getragen werden. Die dazu erforderliche Begründung kann mit „Untersuchung des Herzens in Ruhe und zusätzlich mit pharmakologischem Stress“ angegeben werden (vgl. dazu auch die Empfehlung zur Steigerung bei Untersuchung beider Kniegelenke DER RADIOLOGE 10/2005, Seite M164 ff).

Für die Pulsoximetrie gilt, dass nach den Vorgaben der Strahlenschutzkommission (SSK) bei Patienten, die oberhalb des Richtwertes exponiert werden, eine Überwachung "grundsätzlich angezeigt" ist. Bei Patienten mit eingeschränkter Thermosensorik oder erhöhter Empfindlichkeit gegenüber Erwärmung, Säuglingen und Kleinkindern, Schwangeren, Patienten mit hochgradigen Durchblutungsstörungen (kardial und zerebral), Patienten in extrem schlechten Allgemeinzustand beziehungsweise kachektische Patienten, sedierte oder anästhesierte Patienten, Patienten mit Anfallsleiden, komatöse und delirante Patienten ist danach die Überwachung zwingend (siehe dazu auch DER RADIOLOGE 4-2009, Seite 381). Diese letzteren Voraussetzungen dürften bei Herzuntersuchungen unter Stress in der Regel vorliegen.

In diesen Fällen ist zu empfehlen, im Befund beziehungsweise auch in der Rechnung auf diese Indikationen hinzuweisen. Damit kann im Zweifel Einwendungen gegen den
Ansatz der GOP 650 ff., 602 leichter begegnet werden. Wenn Brück im GOÄ-Kommentar für die Abrechenbarkeit des EKG stets dessen „Aufschrieb“ voraussetzt (siehe Kommentar zu GOP 605), werden offensichtlich diese Besonderheiten der Herzuntersuchung nicht berücksichtigt (und sind offensichtlich auch nicht bekannt), sodass das nicht ausschlaggebend sein kann. Letztlich kann das jedoch dahinstehen, da sich die Dokumentation jedenfalls zu Beweiszwecken empfehlen dürfte.

Zu den Voraussetzungen der Abrechenbarkeit der Grundziffern GOP 1, 5, 75 allgemein verweisen wir auf die Abrechnungsempfehlungen des BDR mit der Bundesärztekammer (siehe DER RADIOLOGE 1 - 2006 Seite M8f.), bei der Herzuntersuchung dürften die Voraussetzung der GOP 1 und 75 ebenfalls in der Regel vorliegen.

\section{Computertomographie des Herzens mit Kontrastmittel}

\begin{tabular}{|l|l|}
\hline $\begin{array}{l}\text { GOÄ } \\
\mathbf{N r} .\end{array}$ & $\begin{array}{l}\text { Beschreibung (nicht Text der } \\
\text { Leistungslegend) }\end{array}$ \\
\hline 1 & Beratung \\
\hline 5371 & CT im Thoraxbereich \\
\hline 5376 & Ergänzende Tomografien \\
\hline 346 & KM Einbringung Hochdruckinjektion \\
\hline 347 & Jede weitere Hochdruckinjektion \\
\hline 650 & EKG \\
\hline 5377 & Computergestützte Analyse \\
\hline 75 & Ausführlicher Befundbericht \\
\hline Optional & \\
\hline 5 & Symptombezogene Untersuchung \\
\hline 602 & (Puls-) Oxymetrie \\
\hline 60 & konsiliarische Erörterung \\
\hline
\end{tabular}




\section{MRT Herz (nur Ruhe-Untersuchung)}

\begin{tabular}{|l|l|}
\hline $\begin{array}{l}\text { GOÄ } \\
\mathbf{N r} .\end{array}$ & $\begin{array}{l}\text { Beschreibung (nicht Text der } \\
\text { Leistungslegend) }\end{array}$ \\
\hline 1 & Beratung \\
\hline 5715 & MRT Thorax \\
\hline 5731 & Ergänzende Serien \\
\hline 346 & KM Einbringung Hochdruckinjektion \\
\hline 347 & Jede weitere Hochdruckinjektion \\
\hline 650 & EKG \\
\hline 5733 & Computergestützte Analyse \\
\hline 75 & Ausführlicher Befundbericht \\
\hline Optional & \\
\hline 5 & Symptombezogene Untersuchung \\
\hline 602 & (Puls-) Oxymetrie \\
\hline 60 & konsiliarische Erörterung \\
\hline
\end{tabular}

MRT Herz (Stress-Perfusions-MRT)

\begin{tabular}{|l|l|}
$\begin{array}{l}\text { GOÄ } \\
\text { Nr. }\end{array}$ & $\begin{array}{l}\text { Beschreibung (nicht Text der } \\
\text { Leistungslegend) }\end{array}$ \\
\hline 1 & Beratung \\
\hline 5715 & MRT Thorax \\
\hline 5731 & Ergänzende Serien \\
\hline 346 & KM Einbringung Hochdruckinjektion \\
\hline 347 & Jede weitere Hochdruckinjektion \\
\hline 271 & Intravenöse Infusion (Adenosin) \\
\hline 650 & EKG \\
\hline 5733 & Computergestützte Analyse \\
\hline 75 & Ausführlicher Befundbericht \\
\hline Optional & \\
\hline 5 & Symptombezogene Untersuchung \\
\hline 602 & (Puls-) Oxymetrie \\
\hline 60 & konsiliarische Erörterung \\
\hline
\end{tabular}

Tabellen nach gemeinsamer Empfehlung von BDR und DRG mit AG Herz- und Gefäßdiagnostik und FuNRad

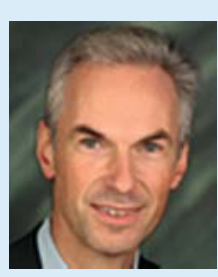

Prof. Meinrad Beer, UIm

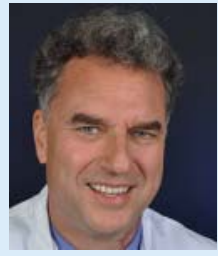

Prof. Stephan Miller, Tübingen

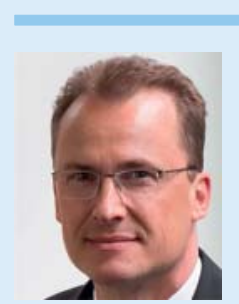

Prof. Jörn Sandstede, Hamburg

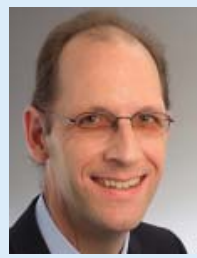
München 